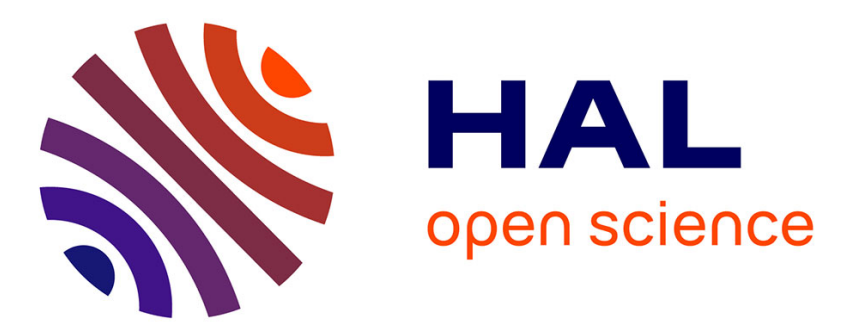

\title{
Modélisation des machines cylindriques asynchrones sans fer. Prise en compte de la longueur finie de la culasse
}

\author{
B. Bandelier, F. Rioux-Damidau
}

\section{To cite this version:}

B. Bandelier, F. Rioux-Damidau. Modélisation des machines cylindriques asynchrones sans fer. Prise en compte de la longueur finie de la culasse. Revue de Physique Appliquée, 1986, 21 (5), pp.327-337. 10.1051/rphysap:01986002105032700 . jpa-00245450

\section{HAL Id: jpa-00245450 https://hal.science/jpa-00245450}

Submitted on 1 Jan 1986

HAL is a multi-disciplinary open access archive for the deposit and dissemination of scientific research documents, whether they are published or not. The documents may come from teaching and research institutions in France or abroad, or from public or private research centers.
L'archive ouverte pluridisciplinaire HAL, est destinée au dépôt et à la diffusion de documents scientifiques de niveau recherche, publiés ou non, émanant des établissements d'enseignement et de recherche français ou étrangers, des laboratoires publics ou privés. 


\title{
Modélisation des machines cylindriques asynchrones sans fer. Prise en compte de la longueur finie de la culasse
}

\author{
B. Bandelier et F. Rioux-Damidau \\ Laboratoire d'Electrotechnique $\left({ }^{*}\right)$ des Universités Paris VI et XI, Bâtiment 214, 91405 Orsay, France
}

(Reçu le 19 novembre 1985, révisé le 27 janvier 1986, accepté le 31 janvier 1986)

\begin{abstract}
Résumé. - Une précédente modélisation à base de séries de Fourier spatio-temporelles avait décrit le système formé du bobinage réel, d'une culasse et d'un rotor infiniment longs. On tient compte des dimensions réelles de la culasse en introduisant les discontinuités de perméabilité directement dans les équations de Maxwell. On obtient des équations relativement complexes et l'on donne une méthode en plusieurs étapes pour les résoudre par simple inversion de matrices.
\end{abstract}

\begin{abstract}
A previous modelling using a double space associated with a temporal Fourier analysis described the device constituted of the real winding and of a yoke and a rotor of infinite lengths. The real dimensions of the yoke are taken into account by directly introducing the permeability discontinuities into the Maxwell's equations. Relatively complex equations are obtained and the method given permits to solve them in several steps by simply inverting matrices.
\end{abstract}

\section{Introduction.}

Une méthode de calcul à base de séries de Fourier et de fonctions de Bessel a été mise au point pour modéliser les machines cylindriques asynchrones sans fer[1]. Elle étend à trois dimensions les techniques utilisées antérieurement en mono- et bi-dimensionnel pour l'étude des moteurs linéaires [2-6]. Cette méthode, du type analytique soutenue par ordinateur, a permis de calculer les champs dans un système simplifié qui comportait :

- le bobinage réel, multipolaire en cuivre,

- un rotor et une culasse magnétique que l'on avait supposés très longs.

Permettant le découplage des différents harmoniques, cette modélisation s'est révélée conduire à des temps de calcul particulièrement performants.

Depuis, une étude tenant compte de la longueur réelle du rotor a été publiée [7]. Elle utilise le même principe de modélisation en imposant artificiellement que le courant soit nul dans les régions où l'on avait supposé précédemment que le rotor se prolongeait.

Cette méthode a permis de s'affranchir du couplage des harmoniques qui s'introduit lorsque l'on écrit

(*) Unité associée au CNRS no 845. les équations pour un système comportant un rotor de longueur finie. Elle a montré que l'approximation $\mathrm{du}$ rotor très long est en général excellente pour les machines construites habituellement.

Nous allons traiter ici la modélisation d'une machine comportant une culasse de longueur réelle et un rotor de longueur infinie, ce qui nous amène à aborder le problème du couplage des harmoniques. Compte tenu de l'absence de saturation nous supposerons sa perméabilité $\mu_{\mathrm{f}}$ constante. Nous supposerons de plus que sa conductivité est suffisamment faible pour que les courants de Foucault y soient négligeables.

\section{Equations générales.}

Le principe du calcul est le même que celui exposé dans [1]. Nous le supposerons pour l'essentiel connu et ne rappellerons ici que les grandes lignes.

Il s'agit de résoudre les équations de Maxwell pour un système (cf. Fig. 1) formé d'une infinité de machines alignées suivant l'axe $z$, régulièrement espacées et suffisamment éloignées les unes des autres pour ne pas interagir. $k$ étant le nombre d'onde, le pas du système a pour longueur $2 \pi / k$ et comporte deux machines symétriques. Le rotor de rayon $R$, de conductivité $\sigma$ est supposé de longueur infinie. Il tourne à la vitesse angulaire $\Omega$. 


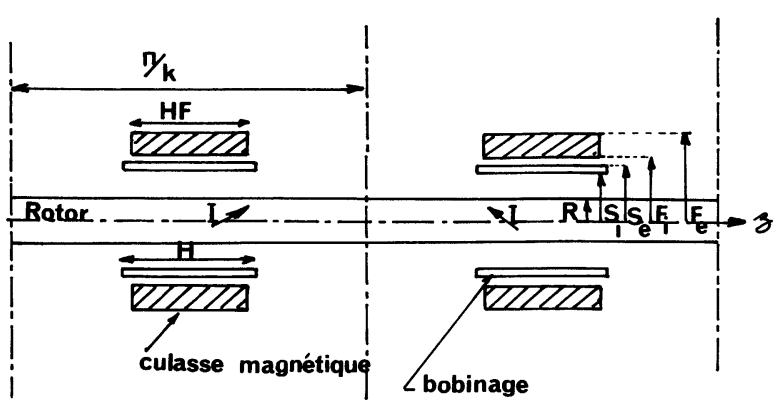

Fig. 1. - Le système modélisé avec rotor infiniment long, bobinage et culasse réels.

[The model with a rotor of infinite length and real winding and yoke.]

Le stator est constitué d'un bobinage polyphasé de longueur $H$, situé entre les rayons $S_{\mathrm{i}}$ et $S_{\mathrm{e}}$. Il est alimenté par un courant de pulsation $\omega$.

La culasse magnétique n'est plus comme dans [1] de longueur et de perméabilité infinies, mais de longueur $H_{\mathrm{F}}$ et de perméabilité $\mu_{\mathrm{f}}$ constante. Elle est située entre les rayons $F_{\mathrm{i}}$ et $F_{\mathrm{e}}$.

Dans le cadre des approximations de l'électrotechnique, les équations de Maxwell s'écrivent :

$$
\begin{aligned}
& \boldsymbol{\nabla} \wedge \mathbf{H}=\mathbf{J} \\
& \boldsymbol{\nabla} \cdot \mathbf{B}=\mathbf{0} \\
& \boldsymbol{\nabla} \wedge \mathbf{E}=-\frac{\partial \mathbf{B}}{\partial t} .
\end{aligned}
$$

L'induction magnétique vérifie :

- dans la culasse

$$
\mathbf{B}=\mu_{\mathrm{f}} \mathbf{H}
$$

— ailleurs

$$
\mathbf{B}=\mu_{0} \mathbf{H} .
$$

Dans [1], on tenait compte de la culasse en écrivant que le champ magnétique était radial au niveau de sa surface intérieure. Pour $\rho<F_{\mathrm{i}}$, l'on avait $\boldsymbol{\nabla} \cdot \mathbf{H}=0$, ce qui permettait d'écrire :

- dans le rotor $(\rho<R)$

$$
\Delta \mathbf{H}=-\sigma \mu_{0}\left(\frac{\partial \mathbf{H}}{\partial \mathbf{t}}+\Omega \frac{\partial \mathbf{H}}{\partial \theta}\right)
$$

$$
\begin{aligned}
&-\operatorname{hors} \operatorname{rotor}\left(R<\rho<F_{\mathrm{i}}\right) \\
& \Delta \mathbf{H}=-\nabla \wedge \mathbf{J} .
\end{aligned}
$$

Dans le cas d'une culasse courte, ces équations restent valables pour $\rho<F_{\mathrm{i}}$. Les différences apparaissent pour $\rho>F_{\mathrm{i}}$. En effet, des discontinuités en $\mu$ interviennent au niveau des extrémités de la culasse où l'on a :

$$
\Delta \mathbf{H}=\boldsymbol{\nabla}(\boldsymbol{\nabla} \cdot \mathbf{H})
$$

avec

$$
\boldsymbol{\nabla} \cdot \mathbf{H}=\boldsymbol{\nabla} \cdot\left(\frac{\mathbf{B}}{\mu}\right)=\nabla\left(\frac{1}{\mu}\right) \cdot \mathbf{B} .
$$

\section{Décomposition en série de Fourier.}

Les équations (3), (4) et (5) se résolvent en écrivant la décomposition en série de Fourier spatio-temporelle des champs et des courants. Pour une grandeur $\mathcal{G}$ on a :

$$
\mathcal{S}(\rho, \theta, z, t)=\sum_{n, l, q \in \mathbf{Z}} G_{n, l, q}(\rho) \mathrm{e}^{i(n \omega t+l \theta+q k z)} .
$$

En remplaçant dans (3), (4), (5) $\mathbf{H}$ et $\mathbf{J}$ par leur décomposition et en intégrant en $t, \theta$ et $z$ sur une période relative à chacune de ces variables (voir [1] et [2]) on obtient les équations d'évolution des modes pour

$$
\begin{aligned}
& H_{1 n, l, q}(\rho)=H_{z n, l, q}(\rho) \\
& H_{2 n, l, q}(\rho)=\left(H_{\rho}-i H_{\theta}\right)_{n, l, q}(\rho)=H_{3-n,-l,-q}^{*}(\rho) .
\end{aligned}
$$

\section{Rappel des résultats de la modélisation présentée dans [1].}

Cette modélisation suppose le rotor et la culasse magnétique infiniment longs. Les équations d'évolution qui sont des équations de Bessel, sans second membre à l'intérieur du rotor, avec second membre à l'extérieur, ne font intervenir qu'une seule valeur de $n, l$ et $q$ à la fois.

La linéarité des équations permet d'exprimer chaque mode sous la forme $\mathbf{H}_{n l q}=I_{n} \cdot \mathbf{h}_{n l q}$. A la différence de [1], nous écrirons les expressions pour les harmoniques $h_{n l q}$, ce qui donne :

- Dans le rotor

$\operatorname{avec} b^{2}=-\left[q^{2} k^{2}+i \sigma \mu_{0}(n \omega+l \Omega)\right]$.

$$
\begin{aligned}
& h_{1 n l q}(\rho)=A_{1 n l q} J_{l}(b \rho) \\
& h_{2 n l q}(\rho)=A_{2 n l q} J_{l-1}(b \rho) \\
& h_{3 n l q}(\rho)=A_{3 n l q} J_{l+1}(b \rho)
\end{aligned}
$$


- Hors rotor

$$
\begin{aligned}
& h_{1 n l q}(\rho)=\left(G_{1 n l q}+C_{2 l q}(\rho)\right) J_{l}(a \rho)+\left(\widetilde{G}_{1 n l q}+\widetilde{C}_{2 l q}(\rho)\right) Y_{l}(a \rho) \\
& h_{2 n l q}(\rho)=\left(G_{1 n l q}+C_{2 l q}(\rho)\right) J_{l-1}(a \rho)+\left(\widetilde{G}_{1 n l q}+\widetilde{C}_{2 l q}(\rho)\right) Y_{l-1}(a \rho) \\
& h_{3 n l q}(\rho)=-\left(G_{1 n l q}+C_{2 l q}(\rho)\right) J_{l+1}(a \rho)-\left(\widetilde{G}_{1 n l q}+\widetilde{C}_{2 l q}(\rho)\right) Y_{l+1}(a \rho)-\left(u_{2 l q}+u_{3 l q}\right) / q k
\end{aligned}
$$

avec $a^{2}=-q^{2} k^{2}$.

Les cinq constantes $A_{1}, A_{2}, A_{3}, G_{1}$ et $\widetilde{G}_{1}$ étant déterminées pour chaque triplet $n, l$ et $q$ par le système linéaire (61) de [1]. $C_{2 l q}(\rho)$ et $\widetilde{C}_{2 l q}(\rho)$ sont des intégrales calculées dans [1].

Elles valent en posant $Z=a \rho=i q k \rho$

$$
\begin{aligned}
& C_{2 l q}(\rho)=\frac{\pi}{2 q k} \int_{a R}^{a \rho}\left(u_{1 l q} Y_{l}(z)-u_{2 l q} Y_{l-1}(z)\right) Z \mathrm{~d} Z \\
& C_{2 l q}(\rho)=-\frac{\pi}{2 q k} \int_{a Z}^{a \rho}\left(u_{1 l q} J_{l}(z)-u_{2 l q} J_{l-1}(z)\right) Z \mathrm{~d} Z
\end{aligned}
$$

$\mathbf{u}_{l q}$ exprime la décomposition en série de Fourier du bobinage.

5. Modélisation d'une culasse magnétique réelle.

5. 1 DESCRIPTION DES DISCONTINUITÉS DE $\mu$. - La différence avec la modélisation d'une culasse de longueur infinie est due au terme $\nabla(1 / \mu)$ qui apparaît dans (6). Si $S$ est la surface de transition air-fer on a (cf. Fig. 2) :

$$
\nabla\left(\frac{1}{\mu}\right) \cdot \mathbf{B}=\left(\frac{1}{\mu_{\mathrm{f}}}-\frac{1}{\mu_{0}}\right)\left(\mathbf{B} \cdot \mathbf{n}_{\mathbf{s}}\right) \delta_{\mathrm{s}}
$$

où $\delta_{S}$ est la diștribution de Dirac [3] et $\mathbf{n}_{S}$ la normale à la surface $S$ orientée dans le sens air-fer. $\mathbf{B} \cdot \mathbf{n}_{S}$ étant continue, l'expression (12) représente le saut que subit le champ normal $\mathbf{H} \cdot \mathbf{n}$ à la traversée de la surface $S$.

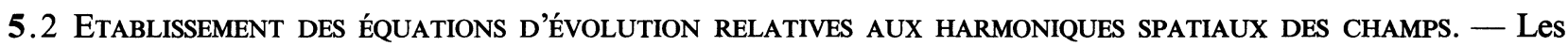
équations (5) et (6) donnent :

$$
\Delta \mathbf{H}=\nabla\left(\nabla\left(\frac{1}{\mu}\right) \cdot \mathbf{B}\right)
$$

et compte tenu de (12)

$$
\Delta \mathbf{H}=\left(\frac{1}{\mu_{\mathrm{f}}}-\frac{1}{\mu_{0}}\right) \nabla\left[\left(\mathbf{B} \cdot \mathbf{n}_{s}\right) \delta_{s}\right]
$$

Dans l'équation (14), nous remplaçons $\mathbf{B}$ et $\mathbf{H}$ par leur décomposition en série de Fourier. En procédant comme au paragraphe 3, il apparaît immédiatement que les équations ne font intervenir qu'une seule valeur de $n$ à la fois. Pour simplifier, nous n'indiquerons plus cette valeur de $n$ que nous supposons connue et nous écrirons $h_{l q}$ au lieu de $h_{n l q} \ldots$ Pour la composante en $z$, il vient ainsi

$$
\begin{aligned}
\left(\frac{\partial^{2}}{\partial \rho^{2}}+\frac{1}{\rho} \frac{\partial}{\partial \rho}-\frac{l^{2}}{\rho^{2}}-q^{2} k^{2}\right) h_{1 l q}(\rho) & -\frac{k}{(2 \pi)^{2}}\left(\frac{1}{\mu_{\mathrm{f}}}-\frac{1}{\mu_{0}}\right) \times \\
& \times \sum_{l^{\prime} q^{\prime}} \int_{0}^{2 \pi} \int_{0}^{2 \pi / k} \frac{\partial}{\partial z}\left(\left(\mathbf{b} \cdot \mathbf{n}_{S}\right)(\rho)_{l{ }^{\prime} q^{\prime}} \mathrm{e}^{i\left(l^{\prime} \theta+q^{\prime} k z\right)} \delta_{S}\right) \mathrm{e}^{-i(l \theta+q k z)} \mathrm{d} \theta \mathrm{d} z=0
\end{aligned}
$$

où $\left(\mathbf{b} \cdot \mathbf{n}_{\mathrm{S}}\right)_{l^{\prime} q^{\prime}}$ représente l'harmonique spatial $l^{\prime} q^{\prime}$ de la composante normale de $\mathbf{B}$.

Dans (15) $\rho$ est fixé. $\delta_{S}$ permet de ne conserver le point $\mathrm{M}$ de coordonnées $\rho, \theta, z$ que s'il appartient à $S$, c'est-à-dire à la surface limite de la culasse qui est formée de trois surfaces dont nous étudierons les contributions séparément. Elles sont définies de la manière suivante (cf. Fig. 3)

$$
\begin{aligned}
& -\rho_{S}=F_{\mathrm{i}} \quad z_{\mathbf{A}} \leqslant z_{S} \leqslant z_{\mathbf{B}} \quad \text { et } \quad z_{\mathbf{C}} \leqslant z_{S} \leqslant z_{\mathbf{D}} \\
& -\rho_{S}=F_{\mathrm{e}} \quad z_{\mathbf{A}} \leqslant z_{S} \leqslant z_{\mathbf{B}} \text { et } \quad z_{\mathbf{C}} \leqslant z_{S} \leqslant z_{\mathbf{D}} \\
& -F_{\mathrm{i}} \leqslant \rho_{S} \leqslant F_{\mathrm{e}} \quad z_{\mathbf{S}}=z_{\mathbf{A}}, z_{\mathbf{B}}, z_{\mathbf{C}}, z_{\mathbf{D}} .
\end{aligned}
$$




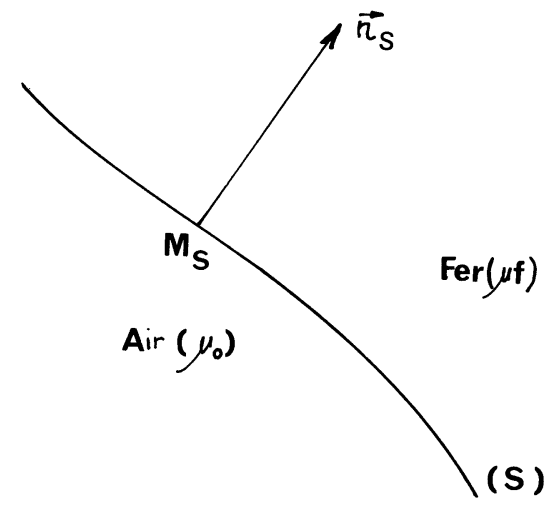

Fig. 2. - Discontinuité entre l'air et le fer.

[The air-iron discontinuity.]

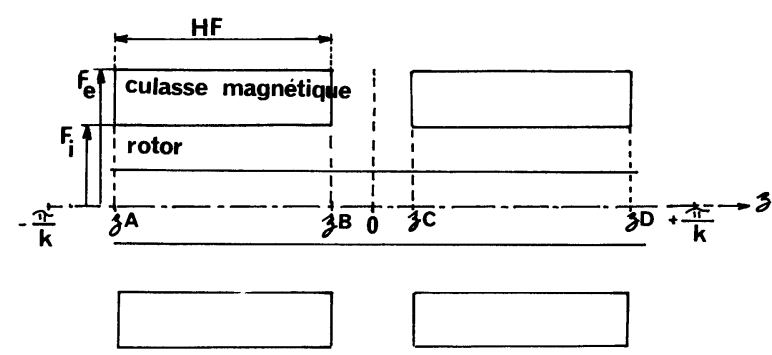

Fig. 3. - Dimensions géométriques de la culasse.

[The yoke geometric dimensions.]

5. 3 CONTRIBUTION DES DIFFÉRENTS TYPES DE SURFACE. - a) Pour une surface cylindrique du type $\rho_{\mathrm{S}}=$ Cte (c'est le cas aux rayons $F_{\mathrm{i}}$ et $F_{\mathrm{e}}$ lorsque la culasse est de longueur infinie) on a :

$$
\begin{gathered}
\rho_{S}=\delta\left(\rho-\rho_{S}\right) . \\
\text { b } \cdot \mathbf{n}_{S}=\varepsilon b_{\rho} \text { avec } \quad \varepsilon=1 \text { si } \rho \text { fer }>\rho \text { air } . \\
\varepsilon=-1 \text { sinon. }
\end{gathered}
$$

L'intégrale de (15) est non nulle seulement pour $l^{\prime}=l$ et $q^{\prime}=q$. Elle s'écrit :

$$
\frac{(2 \pi)^{2}}{k} i q k \varepsilon b_{\rho l q}(\rho) \delta\left(\rho-\rho_{S}\right)
$$

et l'on retrouve le découplage des différents harmoniques observé dans [1]. b) Pour une surface du type $z_{S}=$ Cte (plan perpendiculaire à l'axe) on a :

$$
\begin{aligned}
\delta_{S}=\delta\left(z-z_{S}\right) \\
\mathbf{b} \cdot \mathbf{n}_{S}=\varepsilon b_{1} \quad \text { avec } \quad \varepsilon=1 \quad \text { si } \quad z \text { fer }>z \text { air } \\
\\
\varepsilon=-1 \text { sinon. }
\end{aligned}
$$

D'après les propriétés de la distribution de Dirac [8]

$$
\frac{\partial}{\partial z}\left[\delta\left(z-z_{S}\right)\right] \mathrm{e}^{-i q k z}=i q k \mathrm{e}^{-i q k z} \delta\left(z-z_{S}\right)
$$

et l'intégrale de (15) s'écrit :

$$
2 \pi i q k \sum_{q^{\prime}} \varepsilon b_{1 l q^{\prime}} \mathrm{e}^{i\left(q^{\prime}-q\right) k z_{s}} .
$$

Elle fait intervenir un couplage en $q$ dans l'équation d'évolution des modes $h_{l q}$.

Remarquons que le problème étant indépendant de $\theta$ il y a toujours découplage des harmoniques en $l$.

On pourrait aussi traiter le cas d'une culasse de forme quelconque comportant par exemple des surfaces du type $\theta=$ Cte (fente, encoches). On aurait :

$$
\dot{\delta}_{S}=\dot{\delta}\left(\theta-\theta_{S}\right) \text {. }
$$

L'intégrale de (15) s'écrirait :

$$
\frac{2 \pi}{k} i q k \sum_{l^{\prime}} \varepsilon b_{\theta l^{\prime} q} \mathrm{e}^{i\left(l^{\prime}-l\right) \theta_{s}} .
$$

Il y aurait alors couplage des harmoniques en $l$.

5.4 Equations D'Évolution POUR UNE CULASSE MAGNÉTIQUE RÉELLE. - Ecrivons maintenant les équations pour une culasse cylindrique de rayon intérieur $F_{\mathrm{i}}$, de rayon extérieur $F_{\mathrm{e}}$, de longueur $H_{\mathrm{F}}$ (la culasse de longueur infinie serait telle que $\left.H_{\mathrm{F}}=\pi / k\right)$. La surface limite est définie par (16).

5.4.1 Influence des surfaces interne et externe. Pour décrire la surface interne $\left(\rho_{S}=F_{\mathrm{i}}\right)$ et externe $\left(\rho_{S}=F_{\mathrm{e}}\right)$ de la culasse, nous posons :

$$
\begin{aligned}
& \alpha(z)=1 \text { pour } z_{\mathrm{A}} \leqslant z \leqslant z_{\mathrm{B}} \text { et } z_{\mathrm{C}} \leqslant z \leqslant z_{\mathrm{D}} \\
& \alpha(z)=0 \text { ailleurs. }
\end{aligned}
$$

Dans le cas de la surface interne de la culasse on a :

$$
\delta_{\mathrm{S}}=\delta\left(\rho-F_{\mathrm{i}}\right) \alpha(z) .
$$

L'intégrale de (15) devient, en remplaçant $\alpha(z)$ par sa décomposition en série de Fourier

$$
\sum_{q^{\prime} q^{\prime \prime} \in \mathbb{Z}} 2 \pi \delta\left(\rho-F_{\mathrm{i}}\right) \int_{0}^{2 \pi / k} i\left(q^{\prime}+q^{\prime \prime}\right) k\left[b_{\rho q^{\prime}}(\rho) \alpha_{q^{\prime \prime}}\right] \mathrm{e}^{i\left(q^{\prime}+q^{\prime \prime}-q\right) k z} \mathrm{~d} z
$$

qui est non nul seulement si $q^{\prime}+q^{\prime \prime}=q$. 
En regroupant les termes $q^{\prime}>0$ et $q^{\prime}<0$, il reste compte tenu de la symétrie $\left(b_{\rho l-q^{\prime}}=-b_{\rho l q^{\prime}}\right)$

$$
\sum_{q^{\prime} \in \mathbb{N}} \frac{4 \pi^{2}}{k} i q k b_{\rho l q^{\prime}}(\rho) \alpha_{q q^{\prime}} \delta\left(\rho-F_{\mathrm{i}}\right)
$$

avec

et

$$
\begin{gathered}
\alpha_{q q^{\prime}}=\alpha_{q-q^{\prime}}-\alpha_{q+q^{\prime}}=\frac{2}{\pi}(-1)^{\left(q^{\prime}-q\right) / 2}\left\{\frac{\sin \left(q-q^{\prime}\right) k H_{\mathrm{F}} / 2}{q-q^{\prime}}+\frac{\sin \left(q+q^{\prime}\right) k H_{\mathrm{F}} / 2}{q+q^{\prime}}\right\} \\
\alpha_{q q}=\frac{1}{\pi}\left(k H_{\mathrm{F}}+\frac{\sin q k H_{\mathrm{F}}}{q}\right) .
\end{gathered}
$$

Pour la surface externe de la culasse $\left(\rho_{S}=F_{\mathrm{e}}\right)$ les équations sont similaires à celles obtenues pour $\rho=F_{\mathrm{i}}$. L'intégrale de (15) se déduit directement de l'expression (19) en remplaçant $F_{\mathrm{i}}$ par $F_{\mathrm{e}}$ et en changeant le signe car la normale est dans le sens air-fer. On obtient :

$$
-\sum_{q^{\prime} \in \mathbb{N}} \frac{4 \pi^{2}}{k} i q k b_{\rho l q^{\prime}}(\rho) \alpha_{q q^{\prime}} \delta\left(\rho-F_{\mathrm{e}}\right) .
$$

Remarquons que les expressions (19) et (21) introduisent un couplage des harmoniques en $q$. En revanche, les modes angulaires (en $l$ ) restent toujours découplés.

5.4.2 Contribution des surfaces limites aux extrémités de la culasse. - Les surfaces limites aux extrémités de la culasse sont du type $: z_{\mathrm{S}}=\mathrm{Cte}, F_{\mathrm{i}} \leqslant \rho \leqslant F_{\mathrm{e}}$.

On utilise donc l'équation (18) avec $z_{S}=z_{\mathbf{A}}, z_{\mathbf{B}}, z_{\mathbf{C}}, z_{\mathbf{D}}$ (Fig. 3), $\varepsilon$ valant alternativement +1 et -1 . Pour ces surfaces, l'intégrale de (15) s'écrit :

$$
2 \pi i q k \sum_{q^{\prime}} b_{1 l q^{\prime}}(\rho)\left[\mathrm{e}^{i\left(q^{\prime}-q\right) k z_{\mathbf{A}}}-\mathrm{e}^{i\left(q^{\prime}-q\right) k z_{\mathbf{B}}}+\mathrm{e}^{i\left(q^{\prime}-q\right) k z_{\mathbf{C}}}-\mathrm{e}^{i\left(q^{\prime}-q\right) k z_{\mathrm{D}}}\right] .
$$

Compte tenu de ce que $q$ et $q^{\prime}$ sont impairs [9] on obtient en remplaçant $z_{\mathbf{A}}, z_{\mathbf{B}}, z_{\mathbf{C}}$ et $z_{\mathbf{D}}$ par leurs valeurs :

$$
8 \pi q k \sum_{q^{\prime} \in \mathrm{Z}}(-1)^{\frac{q^{\prime}-q}{2}}: b_{1 l q^{\prime}}(\rho) \cdot \sin \left(q^{\prime}-q\right) \frac{k H_{\mathrm{F}}}{2} .
$$

Vu la symétrie du système, $b_{1 l q}=b_{1 l-q}$ et on obtient finalement :

$$
16 \pi q k \sum_{q^{\prime} \in \mathbb{N}} b_{1 l q^{\prime}}(\rho)(-1)^{\frac{q^{\prime}-q}{2}} \sin q^{\prime} \frac{k H_{\mathrm{F}}}{2} \cos q \frac{k H_{\mathrm{F}}}{2}
$$
en $q$.

Notons que ce terme qui n'existe que pour $F_{\mathrm{i}} \leqslant \rho \leqslant F_{\mathrm{e}}$ introduit également un couplage des seuls modes

5.4.3 Contribution de l'ensemble des surfaces limites de la culasse. - Au total, les contributions à l'intégrale de (15) des différentes surfaces limites de la culasse sont données par (19), (21) et (23). Comme nous l'avons souligné, les modes en $l$ sont découplés. Aussi nous considérerons les équations correspondant à une valeur de l fixée et nous écrirons seulement les harmoniques sous la forme $h_{q}$ (au lieu de $h_{n l q}$ ou $h_{l q}$ comme nous le faisions jusque-là puisque nous avions fixé $n$ ). En regroupant les contributions des différentes surfaces dans (15) on obtient

$$
\begin{array}{r}
\left(\frac{\partial^{2}}{\partial \rho^{2}}+\frac{1}{\rho} \frac{\partial}{\partial \rho}-\frac{l^{2}}{\rho^{2}}-q^{2} k^{2}\right) h_{1 q}(\rho)-\frac{k}{(2 \pi)^{2}}\left(\frac{1}{\mu_{\mathrm{f}}}-\frac{1}{\mu_{0}}\right)\left\{\sum_{q^{\prime} \in \mathrm{N}} \frac{4 \pi^{2}}{k} i q k b_{\rho q^{\prime}}(\rho) \alpha_{q q^{\prime}}\left[\delta\left(\rho-F_{\mathrm{i}}\right)-\delta\left(\rho-F_{\mathrm{e}}\right)\right]+\right. \\
\left.+16 \pi q k \sum_{q^{\prime} \in \mathrm{N}} b_{1 q^{\prime}}(\rho) \cdot(-1)^{\frac{q^{\prime}-q}{2}} \sin q^{\prime} \frac{k H_{\mathrm{F}}}{2} \cos q \frac{k H_{\mathrm{F}}}{2}\right\}=0
\end{array}
$$

Posons comme dans (23) de [1] $Z=a \rho=i q k \rho$

$$
h^{\prime}=\frac{\partial h}{\partial Z}
$$


et de plus

$$
\begin{aligned}
V_{q}=\left(\frac{1}{\mu_{0}}-\frac{1}{\mu_{\mathrm{f}}}\right) \frac{1}{q k}\left[\frac{i}{2}\left(\delta\left(\rho-F_{\mathrm{i}}\right)-\delta\left(\rho-F_{\mathrm{e}}\right)\right) \sum_{q^{\prime} \in \mathbb{N}}\left(b_{2 q^{\prime}}+b_{3 q^{\prime}}\right)(\rho) \alpha_{q q^{\prime}}+\right. \\
\left.+\frac{4 k}{\pi} \cos q \frac{k H_{\mathrm{F}}}{2} \sum_{q^{\prime} \in \mathbb{N}}(-1)^{\frac{q^{\prime}-q}{2}} b_{1 q^{\prime}}(\rho) \sin q^{\prime} \frac{k H_{\mathrm{F}}}{2}\right]
\end{aligned}
$$

On obtient alors

$$
h_{1 q}^{\prime \prime}+\frac{1}{Z} h_{1 q}^{\prime}+\left(1-\frac{l^{2}}{Z^{2}}\right) h_{1 q}=V_{q}
$$

Pour obtenir l'équation d'évolution de $h_{2 q}\left(h_{3}\right)$ on additionne les équations relatives à $h_{\rho q}$ et à $-i h_{\theta q}\left(+i h_{\theta}\right)$. Dans les intégrales équivalentes à celle de(15), apparaissent $\frac{\partial}{\partial \rho}$ et $-\frac{i}{\rho} \frac{\partial}{\partial \theta}\left(+\frac{i}{\rho} \frac{\partial}{\partial \theta}\right)$ à la place de $\frac{\partial}{\partial z}$. On obtient finalement :

$$
\begin{aligned}
& h_{2 q}^{\prime \prime}+\frac{1}{Z} h_{2 q}^{\prime}+\left[1-\left(\frac{l-1}{Z}\right)^{2}\right] h_{2 q}=\frac{l}{Z} V_{q}+V_{q}^{\prime} \\
& h_{3 q}^{\prime \prime}+\frac{1}{Z} h_{3 q}^{\prime}+\left[1-\left(\frac{l+1}{Z}\right)^{2}\right] h_{3 q}=-\frac{l}{Z} V_{q}+V_{q}^{\prime}
\end{aligned}
$$

Les équations d'évolution du champ magnétique (26), (27) et (28) couplent donc par l'intermédiaire de $V_{q}$ les différents modes en $q$.

5.5. EXPRESSIONS DES CHAMPS. - Dans la région du rotor les champs sont toujours donnés par les expressions (9) et pour $R \leqslant \rho \leqslant F_{\mathrm{i}}$ par les expressions (10).

Pour $\rho \geqslant F_{\mathrm{i}}$ ils sont déterminés par les équations (26), (27) et (28). Formellement ce sont des équations de Bessel qui ont la même forme apparente que les équations (30), (31) et (32) de [1] dont les seconds membres étaient $T_{1}, T_{2}, T_{3}$. Mais ici, le second membre est inconnu puisque $V_{q}$ dépend des $b_{q}$. En traitant $V_{q}$ comme s'il était connu on peut écrire les champs.

$$
\begin{aligned}
& h_{1 q}=\left(G_{1 q}+C_{2 q}+F_{1 q}(\rho)\right) J_{l}(a \rho)+\left(\widetilde{G}_{1 q}+\widetilde{C}_{2 q}+\tilde{F}_{1 q}(\rho)\right) Y_{l}(a \rho) \\
& h_{2 q}=\left(G_{1 q}+C_{2 q}+F_{2 q}(\rho)\right) J_{l-1}(a \rho)+\left(\widetilde{G}_{1 q}+\widetilde{C}_{2 q}+\widetilde{F}_{2 q}(\rho)\right) Y_{l-1}(a \rho) \\
& h_{3 q}=\left(-G_{1 q}-C_{2 q}+F_{3 q}(\rho)\right) J_{l+1}(a \rho)+\left(-\widetilde{G}_{1 q}-\widetilde{C}_{2 q}+\widetilde{F}_{3 q}(\rho)\right) Y_{l+1}(a \rho) .
\end{aligned}
$$

$G_{1 q}$ et $\tilde{G}_{1 q}$ sont des constantes. Les intégrales $C_{2 q}$ et $\tilde{C}_{2 q}$ liées à un second membre du type de $T_{1}, T_{2}$ et $T_{3}$ sont toujours données par (11).

$F_{1 q}, F_{2 q}, F_{3 q}$ et $\widetilde{F}_{1 q}, \widetilde{F}_{2 q}, \widetilde{F}_{3 q}$ sont des intégrales liées aux seconds membres de (26), (27) et (28).

$$
\begin{aligned}
& F_{1 q}(a \rho)=-\frac{\pi}{2} \int_{a F_{\overline{1}}^{-}}^{a \rho} V_{q} Y_{l}(Z) Z \mathrm{~d} Z \\
& F_{2 q}(a \rho)=-\frac{\pi}{2} \int_{a F_{\overline{1}}^{-}}^{a \rho}\left(\frac{l}{Z} V_{q}+V_{q}^{\prime}\right) Y_{l-1}(Z) Z \mathrm{~d} Z \\
& F_{3 q}(a \rho)=-\frac{\pi}{2} \int_{a F_{\overline{\mathrm{i}}}}^{\rho}\left(-\frac{l}{Z} V_{q}+V_{q}^{\prime}\right) Y_{l+1}(Z) \mathrm{d} Z
\end{aligned}
$$

où $Z=a \rho=i q k \rho$.

Les expressions de $\widetilde{F}_{1}, \widetilde{F}_{2}$ et $\widetilde{F}_{3}$ s'obtiennent en remplaçant $Y$ par $J$ dans celles de $F_{1}, F_{2}, F_{3}$ et en changeant le signe.

Considérons l'intégrale $F_{2 q}(\rho)$. Elle peut s'écrire :

$$
F_{2 q}(a \rho)=-\frac{\pi}{2} \int_{a F_{\overline{\mathrm{i}}}^{-}}^{a \rho}\left[(l-1) V_{q} Y_{l-1}+\left(Z Y_{l-1} V_{q}\right)^{\prime}-Z Y_{l-1} V_{l q}\right] \mathrm{d} Z
$$


soit

$$
F_{2 q}(a \rho)=-\frac{\pi}{2}\left[Z Y_{l-1} V_{q}\right]_{a F_{\overline{1}}}^{a \rho}+F_{1 q}(a \rho)
$$

De même :

$$
\tilde{F}_{2 q}(a \rho)=+\frac{\pi}{2}\left[Z J_{t-1} V_{q}\right]_{a F_{\overline{1}}}^{a \rho}+\tilde{F}_{1 q}(a \rho)
$$

De la même manière :

$$
F_{3 q}(a \rho)=-\frac{\pi}{2}\left[Z Y_{l+1} V_{q}\right]_{a F_{\bar{Y}}}^{a \rho}-F_{1 q}(a \rho)
$$

et

$$
\tilde{F}_{3 q}(a \rho)=+\frac{\pi}{2}\left[Z J_{l+1} V_{q}\right]_{a F_{\overline{1}}}^{a \rho}+\tilde{F}_{1 q}(a \rho) .
$$

En remplaçant (31), (32), (33) et (34) dans les expressions des champs (29), les termes tout intégrés s'éliminent et il reste simplement :

$$
\begin{aligned}
& h_{1 q}(\rho)=\left(G_{1 q}+C_{2 q}+F_{1 q}(\rho)\right) J_{l}(a \rho)+\left(\widetilde{G}_{1 q}+\widetilde{C}_{2 q}+\widetilde{F}_{1 q}(\rho)\right) Y_{l}(a \rho) \\
& h_{2 q}(\rho)=\left(G_{1 q}+C_{2 q}+F_{1 q}(\rho)\right) J_{l-1}(a \rho)+\left(\widetilde{G}_{1 q}+\widetilde{C}_{2 q}+\widetilde{F}_{1 q}(\rho)\right) Y_{l-1}(a \rho) \\
& h_{3 q}(\rho)=-\left(G_{1 q}+C_{2 q}+F_{1 q}(\rho)\right) J_{l+1}(a \rho)-\left(G_{1 q}+\widetilde{C}_{2 q}+\widetilde{F}_{1 q}(\rho)\right) Y_{l+1}(a \rho)
\end{aligned}
$$

- Pour $\rho<F_{\mathrm{i}}$ les intégrales $F_{1 q}(\rho)$ et $\widetilde{F}_{1 q}(\rho)$ sont nulles et l'on retrouve les expressions (10).

- Pour $F_{\mathrm{i}} \leqslant \rho \leqslant F_{\mathrm{e}}$ on remplace $V_{q}$ dans (30a) par son expression (25). L'intégrale en $\delta\left(\rho-F_{\mathrm{i}}\right)$ est immédiate et il reste

$$
\begin{array}{r}
F_{1}(a \rho)=\left(\frac{1}{\mu_{0}}-\frac{1}{\mu_{\mathrm{f}}}\right) \cdot\left[\frac{-2}{q} \cos q \frac{k H_{\mathrm{F}}}{2} \int_{a F_{1}}^{\rho} \sum_{q^{\prime} \in \mathrm{N}}(-1)^{\frac{q^{\prime}-a}{2}} \sin q^{\prime} \frac{k H_{\mathrm{F}}}{2} b_{1 q^{\prime}}(\rho) Y_{l}(Z) Z \mathrm{~d} Z+\right. \\
\left.\quad+\frac{i \pi}{4} q k F_{\mathrm{i}} \sum_{q^{\prime} \in \mathrm{N}} \alpha_{q q^{\prime}}\left(b_{2}+b_{3}\right)_{q^{\prime}}\left(F_{\mathrm{i}}\right) Y_{l}\left(a F_{\mathrm{i}}\right)\right]
\end{array}
$$

$\widetilde{F}_{1}$ se déduit directement de (36) en remplaçant $Y_{l}$ par $J_{l}$ et en changeant le signe.

Le premier terme de (36) (celui qui comporte l'intégrale) rend compte de la discontinuité du champ à la transition air-fer aux extrémités de la culasse.

Le second terme exprime la discontinuité à la surface interne de la culasse (au rayon $F_{\mathrm{i}}$ ).

Si on se place au rayon $\rho=F_{\mathrm{i}}+\varepsilon$ (surface interne) seul ce second terme est présent dans $F_{1}$ puisque l'intégrale de (36) est alors nulle. Dans ce cas on constate que :

$$
F_{1}\left(a F_{\mathrm{i}}\right) J_{l}\left(a F_{\mathrm{i}}\right)+\tilde{F}_{1}\left(a F_{\mathrm{i}}\right) Y_{l}\left(a F_{\mathrm{i}}\right)=0
$$

et $h_{1 q}\left(F_{\mathrm{i}}\right)=\left(G_{1}+C_{2}\right) J_{l}\left(a F_{\mathrm{i}}\right)+\left(\tilde{G}_{1}+\tilde{C}_{2}\right) Y_{l}\left(a F_{\mathrm{i}}\right)$ qui est l'expression du champ dans l'air.

La continuité de $h_{1}$ est donc bien assurée au rayon $F_{\mathrm{i}}$. On peut vérifier que celle de $h_{\theta}$ l'est également.

- Pour $\rho \geqslant F_{\mathrm{e}}$ l'expression de $F_{1 q}(\rho)+i \widetilde{F}_{1 q}(\rho)$ devient :

$$
\begin{aligned}
F_{1 q}(\rho)+i \widetilde{F}_{1 q}(\rho)=\left(\frac{1}{\mu_{0}}-\frac{1}{\mu_{\mathrm{f}}}\right) & {\left[-\frac{2}{q} \cos q \frac{k H_{\mathrm{F}}}{2} \int_{a F_{\mathrm{F}}}^{a F_{\mathrm{e}}} \sum_{q^{\prime} \in \mathrm{N}}(-1)^{\frac{q^{\prime}-a}{2}} \sin q^{\prime} \frac{k H_{\mathrm{F}}}{2} b_{1 q^{\prime}}(\rho) \times\right.} \\
& \times\left(Y_{l}(Z)-i J_{l}(Z)\right) Z \mathrm{~d} Z+\frac{i \pi}{4} q k F_{\mathrm{i}} \sum_{q^{\prime} \in \mathrm{N}} \alpha_{q q^{\prime}}\left(b_{2}+b_{3}\right)_{q^{\prime}}\left(F_{\mathrm{i}}\right)\left(Y_{l}\left(a F_{\mathrm{i}}\right)-i J_{l}\left(a F_{\mathrm{i}}\right)\right) \\
& \left.-\frac{i \pi}{4} q k F_{\mathrm{e}} \sum_{q^{\prime} \in \mathrm{N}} \alpha_{q q^{\prime}}\left(b_{2}+b_{3}\right)_{q^{\prime}}\left(F_{\mathrm{e}}\right)\left(Y_{l}\left(a F_{\mathrm{e}}\right)-i J_{l}\left(a F_{\mathrm{e}}\right)\right)\right]
\end{aligned}
$$

où la partie en $Y_{l}$ correspond à $F_{1}$ et celle en $J_{l}$ à $\widetilde{F}_{1}$. 
(37) comporte un terme supplémentaire par rapport à (36) qui exprime la discontinuité du champ à la traversée de la surface extérieure de la culasse (au rayon $F_{\mathrm{e}}$ ).

Notons enfin que $F_{1 q}(\infty)=F_{1 q}\left(F_{\mathrm{e}}\right)$.

Lorsque la culasse est infiniment longue (c'est-à-dire $H_{\mathrm{F}}=\pi / k$ ) le premier terme de (36) (et de (37)) est nul. D'autre part $\alpha_{q q^{\prime}}=\delta_{q q^{\prime}}, \delta$ étant le symbole de Kronecker. Dans ce cas, comme cela était prévisible, le couplage de modes disparaît.

5.6 DÉTERMINATION DES CONSTANTES POUR UNE CULASSE DE LONGUEUR FINIE. - Les champs d'un mode donné dépendent des cinq constantes $A_{1 q}$, $A_{2 q}, A_{3 q}, G_{1 q}$ et $\widetilde{G}_{1 q}$ qui sont déterminées à partir des conditions aux limites.

Les quatre premières équations du système (61) de [1], qui correspondent à des conditions aux limites du rotor et à l'intérieur de celui-ci, restent valables. La cinquième qui considérait que le champ était radial pour $\rho=F_{\mathrm{i}}$ doit être modifiée. Nous devons écrire que les champs sont nuls à l'infini. En supposant qu'il n'y a pas de conducteur au-delà du rayon $S_{e}$, on a :

$$
\begin{aligned}
& C_{2 q}(\infty)=C_{2 q}\left(S_{\mathrm{e}}\right) \\
& \tilde{C}_{2 q}(\infty)=\widetilde{C}_{2 q}\left(S_{\mathrm{e}}\right) .
\end{aligned}
$$

Compte tenu de ce que $F_{1 q}(\infty)=F_{1 q}\left(F_{\mathrm{e}}\right)$ et $\tilde{F}_{1 q}(\infty)=\tilde{F}_{1 q}\left(F_{\mathrm{e}}\right)$, la condition des champs nuls à l'infini s'écrit :

$$
\begin{aligned}
G_{1 q}+C_{2 q}\left(S_{\mathrm{e}}\right) & +F_{1 q}\left(F_{\mathrm{e}}\right)+ \\
+ & i\left[\widetilde{G}_{1 q}+\widetilde{C}_{2 q}\left(S_{\mathrm{e}}\right)+\widetilde{F}_{1 q}\left(F_{\mathrm{e}}\right)\right]=0 .
\end{aligned}
$$

Les équations s'écrivent donc :

$$
\begin{gathered}
2 a A_{1 q}-b A_{2 q}+b A_{3 q}=0 \\
J_{l}(b R) A_{1 q}-J_{l}(a R) G_{1 q}-Y_{l}(a R) \tilde{G}_{1 q}=0 \\
J_{l-1}(b R) A_{2 q}-J_{l-1}(a R) G_{1 q}-Y_{l-1}(a R) \tilde{G}_{1 q}=0 \\
J_{l+1}(b R) A_{3 q}-J_{l+1}(a R) G_{1 q}-Y_{l+1}(a R) \tilde{G}_{1 q}=0 \\
G_{1 q}+i \tilde{G}_{1 q}=-C_{2 q}\left(S_{\mathrm{e}}\right)-i \tilde{C}_{2 q}\left(S_{\mathrm{e}}\right)- \\
-F_{1 q}\left(F_{\mathrm{e}}\right)-i \tilde{F}_{1 q}\left(F_{\mathrm{e}}\right)
\end{gathered}
$$

où $F_{1 q}\left(F_{\mathrm{e}}\right)+i \widetilde{F}_{1}\left(F_{\mathrm{e}}\right)$ est donné par (37).

Ces équations (39) ne forment pas un système indépendant. Par l'intermédiaire de $F_{1 q}$ et $\widetilde{F}_{1 q}$, il apparaît un couplage avec tous les autres systèmes de même type correspondant aux mêmes valeurs de $n$ et $l$ mais à des valeurs de $q$ différentes.

Si la décomposition en série de Fourier se limite à $Q$ harmoniques suivant $z$, on obtient finalement un système de $5 \times Q$ équations à $5 \times Q$ inconnues, mais qui est compliqué puisque $F_{1}$ et $\widetilde{F}_{1}$ dépendent des harmoniques $b_{q}$ des champs.

\section{Méthode de résolution.}

Si nous considérons seulement l'un des sous-systèmes (39), les quatre premières équations permettent de calculer les rapports

$$
\frac{A_{1 q}}{G_{1 q}}, \frac{A_{2 q}}{G_{1 q}}, \frac{A_{3 q}}{G_{1 q}} \text { et } \frac{\tilde{G}_{1 q}}{G_{1 q}}
$$

qui sont indépendants de la culasse. Il ne reste plus que l'équation :

$$
G_{1 q}\left(1+\frac{i \widetilde{G}_{1 q}}{G_{1 q}}\right)=-C_{2 q}-i \widetilde{C}_{2 q}-F_{1 q}-i \widetilde{F}_{1 q}
$$

où $C_{2}+i \widetilde{C}_{2 q}$ est connu.

Dans $F_{1 q}+i \widetilde{F}_{1 q}$ apparaissent les champs $b_{1 q^{\prime}}(\rho)$, $\left(b_{2}+b_{3}\right)_{q^{\prime}}\left(F_{\mathrm{i}}\right)$ et $\left(b_{2}+b_{3}\right)_{q^{\prime}}\left(F_{\mathrm{e}}\right)$. (Cf. (37).)

$b_{1 q^{\prime}}(\rho)$ reste à déterminer.

$\left(b_{2}+b_{3}\right)_{q^{\prime}}\left(F_{\mathrm{i}}\right)$ est la composante normale à la transition air-fer. On peut donc, par continuité l'écrire $\mu_{0}\left(h_{2}+h_{3}\right)_{q^{\prime}}\left(F_{\mathrm{i}}-\varepsilon\right)$, qui s'exprime simplement en fonction des $G_{1 q}$, cf. (10).

$\left(b_{2}+b_{3}\right)_{q^{\prime}}\left(F_{\mathrm{e}}\right)$ ne peut pas être calculé dans l'air au rayon $F_{\mathrm{e}}+\varepsilon$ car le troisième terme de discontinuité en $F_{\mathrm{e}}$ fait intervenir $\left(b_{2}+b_{3}\right)_{q^{\prime}}\left(F_{\mathrm{e}}\right)$ lui-même. On s'affranchit de ce troisième terme en calculant $\left(b_{2}+b_{3}\right)_{q^{\prime}}\left(F_{\mathrm{e}}-\varepsilon\right)$. Comme ce champ est multiplié par $\alpha_{q q^{\prime}}(\mathrm{cf.}$ (37)), il n'intervient que là où la culasse est présente. On peut alors l'écrire sous la forme $\mu_{\mathrm{f}}\left(h_{2}+h_{3}\right)_{q^{\prime}}\left(F_{\mathrm{e}}-\varepsilon\right)$ qui s'exprime d'après (35) et (36) en fonction des $G_{1 q}$ et des $b_{1 q}(\rho)$.

Au total, $F_{1 q}+i \widetilde{F}_{1 q}$ dépend des $G_{1 q}$ et des $b_{1 q}(\rho)$ avec $F_{\mathrm{i}}<\rho<F_{\mathrm{e}}$, et on peut montrer que (40) s'écrit, après discrétisation de l'intégrale de (37) sous la forme :

$$
\begin{aligned}
G_{1 q}\left(1+i \frac{G_{1 q}}{G_{1 q}}\right)=C_{q}+\sum_{q^{\prime}} & \lambda_{q q^{\prime}} G_{1 q^{\prime}}+ \\
& +\sum_{i} \sum_{q^{\prime}} \varphi_{q q^{\prime} i} b_{1 q^{\prime}}\left(\rho_{i}\right) .
\end{aligned}
$$

A chaque valeur de $q$ correspond une équation du type de (42). $C_{q}$ est un terme connu qui dépend des intégrales $C_{2}$ et $\tilde{C}_{2}$.

L'ensemble de ces $Q$ équations forme un système linéaire où les inconnues sont les $G_{1 q}$, mais où les $b_{1 q}\left(\rho_{i}\right)$ sont indéterminés. Sous forme matricielle, il s'écrit :

$$
A G=C+\Lambda G+\sum_{i=1}^{i t} \Phi_{i} B_{1 i}
$$

soit

$$
(A-\Lambda) G=C+\sum_{i=1}^{i t} \Phi_{i} B_{1 i}
$$

où it est le nombre de points d'intégration entre $F_{\mathrm{i}}$ et $F_{\mathrm{e}}$

$G$ est le vecteur des $Q$ inconnues

$B_{1 i}$ est le vecteur dont les $Q$ composantes sont les $b_{1 q}\left(\rho_{i}\right)$, indéterminées. 
Nous n'exprimons pas ces $b_{1 q}\left(\rho_{i}\right)$ en fonction des $h_{1 q}$. En effet le calcul des coefficients de Fourier $(\mu h)_{q}$ pose des problèmes du point de vue numérique car $\mu$ et $h$ sont tous deux discontinus au même point. Pour résoudre (43) nous procéderons en deux temps.

Nous rechercherons d'abord une expression formelle de $G$ en fonction des $B_{1 i}$, puis nous évaluerons les $B_{1 i}$ en écrivant que $h_{1}\left(\rho_{i}\right)=\frac{b_{1}\left(\rho_{i}\right)}{\mu}$.

- Cherchons $G$ sous la forme

$$
G=G_{0}+\sum_{i=1}^{i t} \Gamma_{i} B_{1 i}
$$

En reportant (44) dans (43), on obtient

$$
\begin{aligned}
G_{0} & =(A-\Lambda)^{-1} C \\
\Gamma_{i} & =(A-\Lambda)^{-1} \Phi_{i} .
\end{aligned}
$$

- Pour déterminer $B_{1 i}$ on part de la relation entre $h_{1}\left(\rho_{j}\right)$ et $b_{1}\left(\rho_{j}\right)$ que l'on écrit :

$$
h_{1 q}\left(\rho_{j}\right)=\sum_{q^{\prime}=1}^{\theta} v_{q q^{\prime}} b_{1 q^{\prime}}\left(\rho_{j}\right)
$$

où $v_{q q^{\prime}}$ tient compte du fait que $\mu=\mu_{\mathrm{f}}$ dans la culasse et $\mu_{0}$ à l'extérieur.

Pour $F_{\mathrm{i}}<\rho_{j}<F_{\mathrm{e}}, h_{1 q}\left(\rho_{j}\right)$ est donné par la première des équations (35). Il s'exprime en fonction des $G_{1 q}$ que nous venons de déterminer et qui dépendent des $b_{1 q}\left(\rho_{i}\right)$ (cf. (44), (45) et (46)), et en fonction des $b_{1 q}\left(\rho_{i}\right)$ eux-mêmes. En reportant cette expression de $h_{1 q}\left(\rho_{j}\right)$ dans (47) on obtient un système linéaire de $Q \times i t$ équations qui permet de déterminer les $b_{1 q}\left(\rho_{i}\right)$.

On en tire immédiatement les $G_{1 q}$ par (44) et donc $\widetilde{G}_{1 q}, A_{1 q}, A_{2 q}$ et $A_{3 q}$. On peut alors calculer les coefficients de Fourier des champs à la surface du rotor, dans le bobinage et en déduire les performances de la machine de façon identique à [1].

\section{Modélisation numérique.}

7.1 Organisation du Calcul. - Dans le cas d'une culasse infinie, les coefficients de Fourier des champs sont obtenus en résolvant pour chaque valeur de $n, l$ et $q$ un système de 5 équations à 5 inconnues, le système (61) de [1].

Pour la culasse courte, on résout successivement pour chaque valeur de $n$ et $l$.

1) $Q$ systèmes de 4 équations à 4 inconnues

2) un système de $Q$ équations à $Q$ inconnues (45)

3) un système de $Q \times$ it équations à $Q \times i t$ inconnues correspondant à (47).

Le temps de calcul est directement lié à la taille des matrices considérées, c'est-à-dire au nombre d'harmonique $Q$ suivant $z$ et au nombre it de points d'intégration entre $F_{\mathrm{i}}$ et $F_{\mathrm{e}}$. Il est environ 7 fois plus grand que celui mis en jeu en [1] mais reste encore très court pour une modélisation tridimensionnelle.
7.2 RÉsultats. - Pour tester cette modélisation nous l'avons appliquée à une machine ayant un rotor de $0,25 \mathrm{~m}$ de diamètre et un bobinage hélicoïdal de $1 \mathrm{~m}$ de long et $2 \mathrm{~cm}$ d'épaisseur alimenté par un courant sinusoïdal. Le diamètre intérieur de la culasse est de $0,317 \mathrm{~cm}$.

Nous avons tout d'abord testé l'influence de l'épaisseur de la culasse pour deux valeurs de glissement dans le cas d'une culasse de même longueur que le bobinage, et d'une culasse de longueur moitié. Au-delà d'une épaisseur de 1 à $2 \mathrm{~cm}$ les performances apparaissent pratiquement indépendantes de cette épaisseur (Fig. 4).

Ensuite, nous avons étudié l'influence de la perméabilité de la culasse. Au-delà de $\mu_{\mathrm{f}} / \mu_{0}=100$, les performances sont peu modifiées.
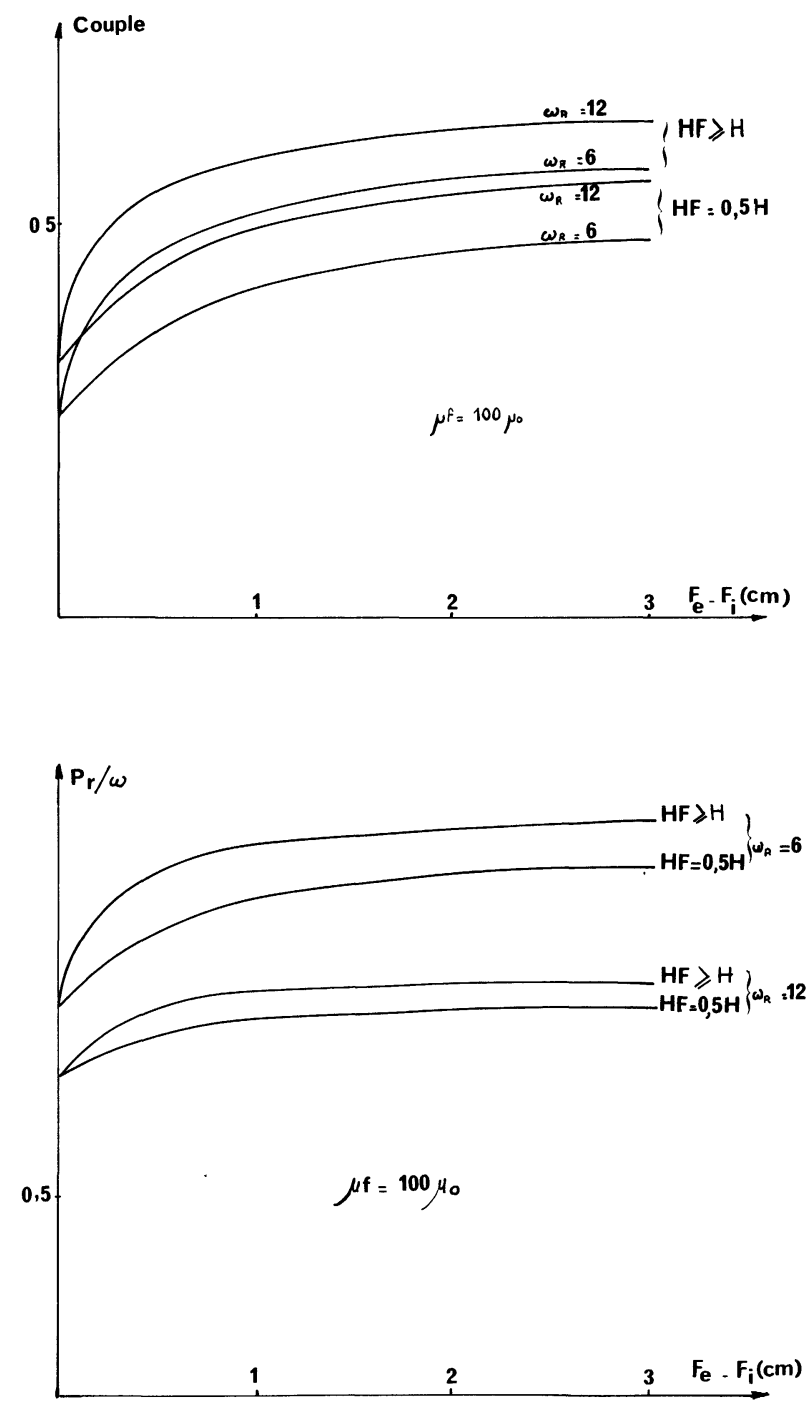

Fig. 4. - Couple et puissance réactive en fonction de l'épaisseur de la culasse ( $\omega_{R}$ est la pulsation rotorique).

[The torque (a) and the reactive power (b) as a function of the yoke thickness ( $\omega_{\mathbf{R}}$ is the rotor angular frequency).] 
Enfin, pour une épaisseur de $3 \mathrm{~cm}$ et $\mu_{\mathrm{f}}=100 \mu_{0}$ nous avons tracé le couple, la puissance réactive et le facteur de puissance en fonction de la pulsation rotorique pour diverses valeurs de la longueur de la culasse. Nous constatons (Fig. 6) que les calculs effectués pour une culasse de longueur infinie donnent pratiquement les mêmes résultats que ceux obtenus avec une culasse de même longueur que le bobinage.

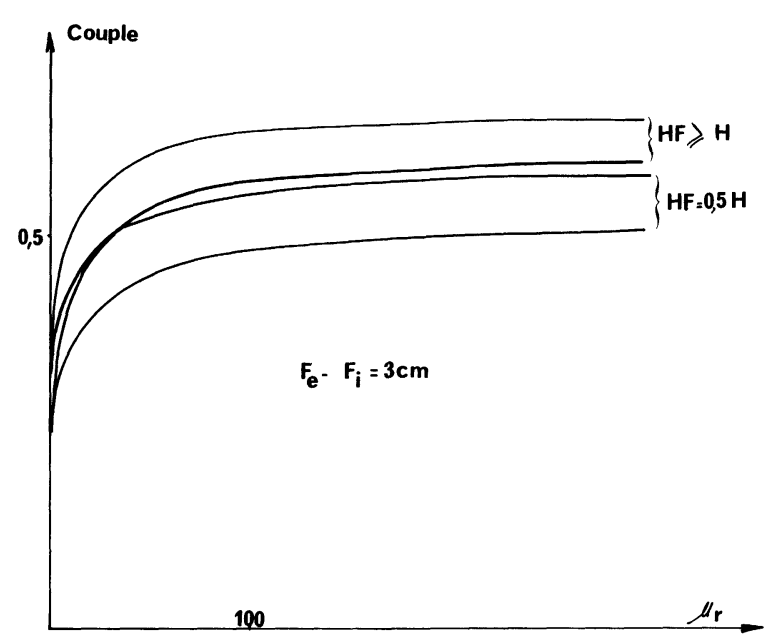

Fig. 5. - Couple et puissance réactive en fonction de la perméabilité relative ( $\omega_{\mathbf{R}}$ est la pulsation rotorique).
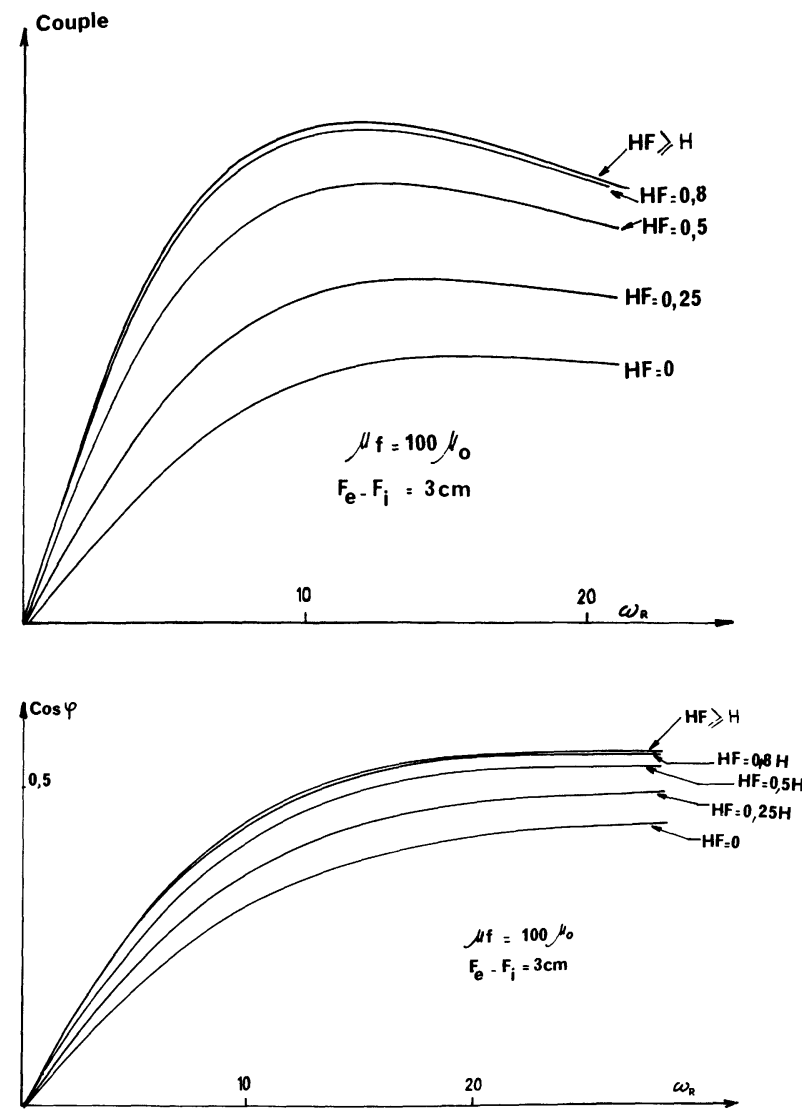

L'influence de la diminution de cette longueur ne commence à être sensible que lorsque $H_{\mathrm{F}} / H$ devient inférieur à 0,8 .

De façon générale, la diminution de la longueur de la culasse réduit le couple, la puissance réactive et le facteur de puissance. De plus, elle déplace légèrement la valeur de la pulsation rotorique donnant le couple maximum vers des valeurs plus élevées.

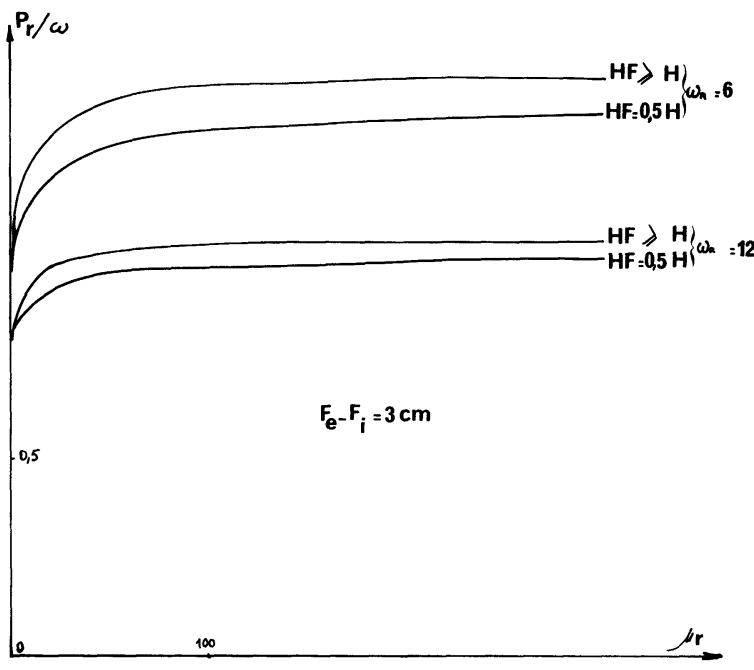

[The torque (a) and the reactive power (b) as a function of the relative permeability ( $\omega_{\mathbf{R}}$ is the rotor angular frequency).]

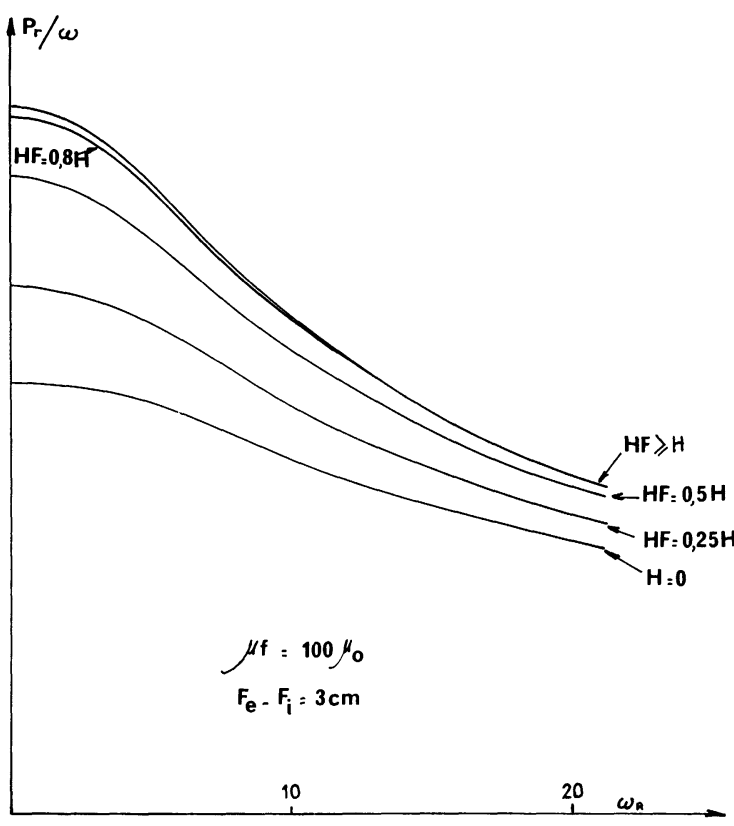

Fig. 6. - Couple, puissance réactive et facteur de puissance en fonction de la pulsation rotorique pour diverses longueurs de culasse.

[The torque (a), the reactive power (b), the power factor (c) as a function of the rotor angular frequency for different yoke lengths.] 


\section{Bibliographie}

[1] Rioux-Damidau, F., Revue Phys. Appl. 18 (1983) 113.

[2] Poloujadoff, M., The theory of linear induction machinery (Oxford University Press) 1980.

[3] Jufer, M., Rev. Gen. Elect. 80 (1971) 108.

[4] Kant, M., Mouillet, A., Scheuer, J. M., Rev. Gen. Elect. 80 (1971) 13.

[5] Sabonnadière, J.C., Poloujadoff, M., Rev. Gen. Elect. 80 (1971) 34.
[6] Isma'eel, M. E., Proc. IEE 125 (1978) 657.

[7] Rioux-Damidau, F., Revue Phys. Appl. 20 (1985) 235.

[8] RodDIER, F., Distributions et transformations de Fourier, (McGraw Hill) 1978.

[9] Rioux-Damidau, F., Bandelier, B., Buret, F., Rev. Gen. Elect. (1985) 203.

[10] Bandelier, B., Thèse de Doctorat, Université Paris VI, Avril 1986. 